ISSN 1112-9867

http://www.jfas.info

\title{
VALORIZATION AGRICULTURAL OF A SOLID DIGESTATE AVICOLOUS RESULTING FROM THE INDUSTRIAL BIOMETHANISATION IN TUNISIA
}

\author{
Y. M'Sadak*, A. Ben M'Barek \\ Institut Supérieur Agronomique de Chott-Mariem, BP 47, CP 4042, \\ Université de Sousse, Tunisie
}

Received: 14 February 2015 / Accepted: 07 July 2015 / Published online: 1 September 2015

\begin{abstract}
The use of the Peat (T) mixed with of methacompost of poultry (MCA) with / without addition of crude forestry compost (CSB) in the substrate of culture prepared has been an experiment in soilless nursery. The growth and survival of tomato plants, among other things, were studied in two experiments, one mixes on MCA $+\mathrm{T}$ and the other on mixtures $\mathrm{CSB}+\mathrm{T}+\mathrm{MCA}$, as compared with the reference substrate $(\mathrm{T})$. The tests performed confirmed that the incorporation of MCA and CSB with T provides slightly better growth in height of tomato plants while limiting bleeding Force by imports of peat substrates. Statistically, there was not observed a significant difference between the height of plants. Height growth of tomato plants was influenced by the type of partial substitute. In this regard, we find that plants grown on substrates mixture have stunted growth due to the apparent phytotoxicity of methacompost of poultry, physical quality and especially its water unsuitable behavior.
\end{abstract}

Keywords: Nursery aboveground vegetable; Methacompost of poultry; Compost forestry; Substrate of culture; Agronomic behavior; Tomato plants.

Author Correspondence, e-mail: msadak.youssef@yahoo.fr doi: http://dx.doi.org/10.4314/jfas.v7i3.1 


\section{INTRODUCTION}

La biomasse représente la seule source des combustibles liquides, solides et gazeux [1], elle est unique, car parmi les énergies renouvelables, elle est la seule qui est constituée de matière organique [2]. La valorisation des déchets agricoles pour la production de biogaz (par digestion anaérobique ou Biométhanisation) pourrait être considérée comme une solution économique, décentralisée et écologique à travers une autonomie énergétique et un développement agricole durable [3]. Les déjections animales sont particulièrement intéressantes à exploiter agronomiquement quand elles sont produites en quantités importantes et régulières $[3,4,5,6,7,8]$ et surtout lorsqu'elles sont traitées par Biométhanisation avant utilisation $[9,10,11]$. La production de biogaz, fondée sur des matières premières produites dans les exploitations agricoles, représente une opportunité intéressante et contribue à répondre aux exigences énergétiques à venir. La production agricole de biogaz s'est développée tout d'abord à partir de l'utilisation de fumier de ferme liquide. Le fumier fût utilisé ainsi dans les installations biogaz du fait des grosses quantités disponibles, de sa grande teneur en matière sèche et de la capacité de formation de gaz [12].

La gestion de la Matière Organique (MO) reste une priorité dans la gestion des déchets. La filière de retour au sol des MO se construit, avec aujourd'hui le développement important de la Biométhanisation [13]. La Biométhanisation permet de transformer la matière organique volatile en énergie, tout en préservant son potentiel fertilisant, aussi bien du point de vue de la matière organique que des éléments minéraux. Ce bioprocédé offre ainsi une solution de valorisation énergétique de la biomasse qui, loin d'être en concurrence avec les impératifs agronomiques, est au contraire en synergie avec ceux-ci [14]. Elle vise à transformer le carbone de la $\mathrm{MO}$ en méthane $\left(\mathrm{CH}_{4}\right)$. La matière résiduelle résultante du procédé, qui peut représenter de 70 à $80 \%$ de la masse initiale est appelée digestat. La valorisation de ce digestat, Co-produit secondaire ou résidu de la Biométhanisation, est essentielle pour rentabiliser cette filière de traitement anaérobie, afin de ne pas créer un nouveau type de déchet [15].

Les Co-produits secondaires ou digestats de la Biométhanisation peuvent être utilisés à l'état solide (Méthacompost) comme partie intégrante des substrats de culture [16] ou être épandus 
$[17,18,19]$, comme ils peuvent être utilisés directement à l'état liquide (Jus de Process) comme fertilisant des sols agricoles [20, 21], voire, en culture hors sol.

La tourbe importée classiquement utilisée dans la confection des substrats horticoles est une ressource non renouvelable $[22,23]$. Il serait intéressant de développer la recherche de produits alternatifs et d'encourager l'emploi partiel des produits de substitution, qui participent à la conservation des avantages de la tourbe et permettent d'en limiter les quantités utilisées, et par conséquent, diminuant l'hémorragie des Devises, conséquente des importations.

Dans ce cadre, la présente investigation constitue une contribution à l'étude qualitative indirecte des substrats de croissance à base de mélange pour une meilleure exploitation en pépinière maraîchère hors sol par la mise en place de deux essais agronomiques dont les objectifs assignés sont respectivement :

- Évaluation de l'incidence de la substitution partielle de la tourbe importée par le MCA dans la confection des substrats de culture pour la production hors sol des plants de tomate.

- Appréciation de l'effet de la substitution partielle de la tourbe importée par l'incorporation du MCA et du CSB dans la confection des substrats de culture pour la production hors sol des plants de tomate.

La tomate étant utilisée dans les différents secteurs de production végétale et a une importance sur le marché économique local. De plus, la littérature confirme l'adaptabilité de cette espèce maraîchère aux techniques de culture hors sol et sa sensibilité aux substances humiques (Herrera et al 2008 cités par [24]).

\section{MATÉRIEL ET MÉTHODES}

\subsection{Site expérimental et digesteur mis en œuvre}

Il s'agit d'un digesteur industriel pilote de forme cylindrique, installé dans une ferme avicole à Hammam Sousse relevant du gouvernorat de Sousse (Tunisie), depuis l'année 2000, d'une capacité utile de $300 \mathrm{~m}^{3}$, alimenté en continu quotidiennement par $10 \mathrm{~m}^{3}$ de substrat composé d'environ $1 / 3$ de fientes avicoles et $2 / 3$ d'eau provenant lors du raclage des poulaillers avec l'effluent sortant décanté (solution aqueuse ou surnageant). L'alimentation en fientes avicoles étant mécanique et le raclage des poulaillers permet une bonne dilution du substrat et un 
pompage facile [25]. Ce digesteur industriel cylindrique, disposé verticalement, est du type digesteur de contact anaérobie à cellules immobilisées (à biomasse fixée). Il est composé d'un milieu réactionnel rempli d'un solide (lit fixe) sur lequel vont se fixer les bactéries. Le garnissage est constitué d'un support inerte de nature (6000 briques de 12). Un tel digesteur fonctionne avec un flux ascendant/descendant. Suivant ce procédé, l'affluent s'écoule vers le haut ou vers le bas (selon sa teneur en solides) à travers le digesteur contenant les briques de 12 qui retiennent les microorganismes anaérobies et sur lesquelles ils se multiplient.

Les fientes digérées, produites dans le digesteur, traversent trois bassins différents (Figure 1) : Un premier bassin recevant le substrat digéré pour un faible temps de séjour (appelé bassin des fientes digérées) avant de séjourner dans le second bassin, appelé bassin de décantation ou décanteur. À partir de ce dernier, les boues des fientes décantées seront séchées et transformées en méthacompost. Quant à l'eau surnageant (Jus de process) le bassin de décantation, elle passe dans le troisième bassin pour servir actuellement au raclage des fientes fraîches des poulaillers. Il convient de signaler que conjointement à chaque opération d'alimentation par de nouvelles fientes, une même quantité de fientes traitées s'évacue à partir du trop plein vers le bassin de collecte des fientes digérées.

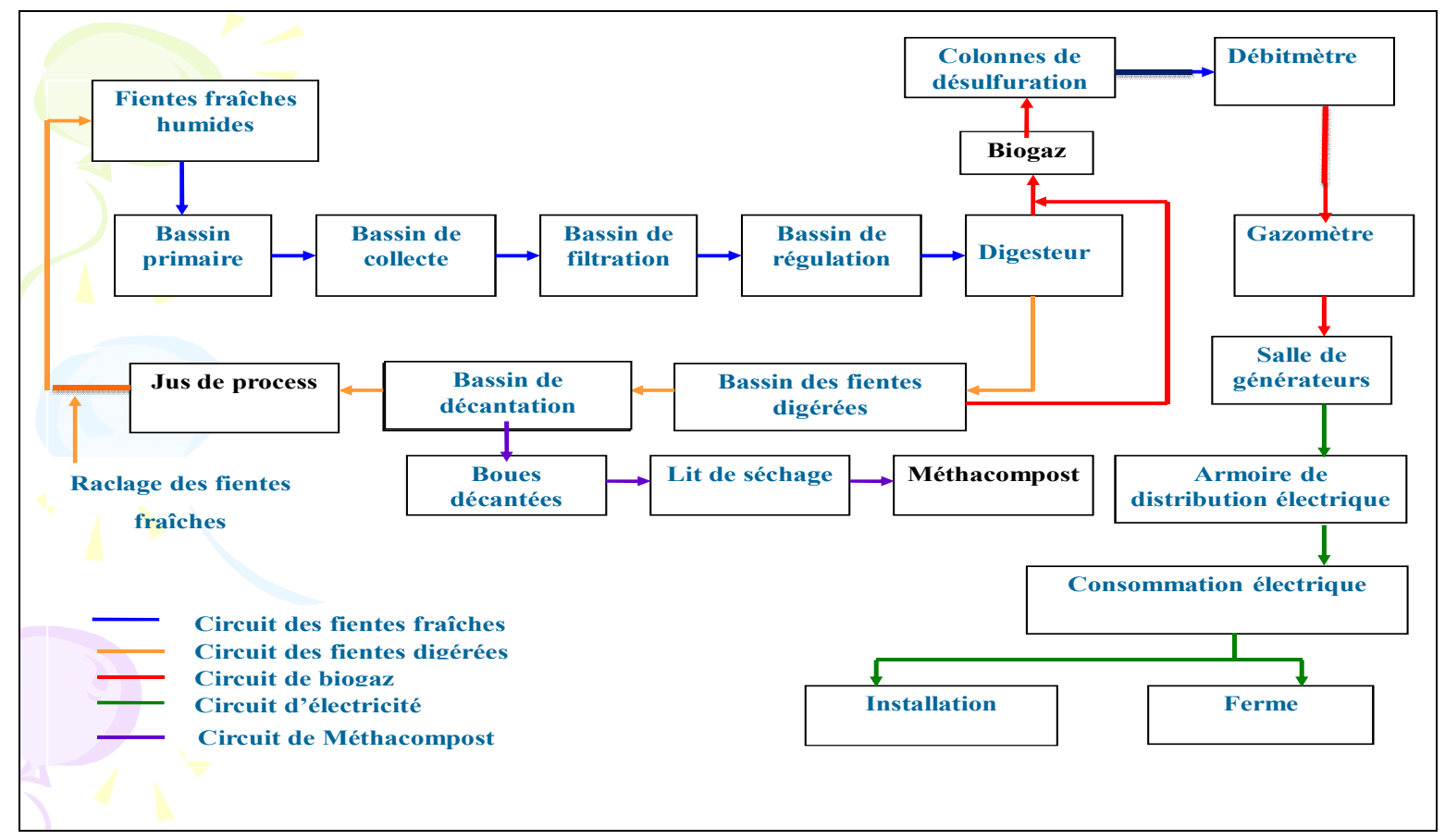

Fig.1. Schéma synoptique des circuits de la Biométhanisation industrielle avicole adoptée 


\subsection{Substrats de croissance mis à l'essai}

Le choix du substrat dans la production maraîchère hors sol est très important. Présentement, la tourbe est la principale composante des substrats utilisés en pépinière grâce à ses nombreuses caractéristiques intéressantes. Elle possède une rétention très élevée, c'est pourquoi d'autres matières y sont généralement ajoutées en mélange.

À partir de trois produits purs considérés, on a réalisé neuf mélanges, tout en substituant partiellement la tourbe par le MCA avec/sans intégration du CSB (\% volume). On a utilisé différentes combinaisons de tourbe, du MCA et du CSB pour voir si le MCA pourrait réellement être un substitut de la tourbe. Les mélanges testés ont été répartis en deux séries relatées dans le Tableau 1. Il s'agit de déterminer les mélanges les plus adéquats, pouvant être employés comme substrats de culture pour la production des plants maraîchers en pépinière hors sol, en les comparant avec les résultats obtenus pour la tourbe utilisée comme support de référence. Au total, il s'agit de dix substrats de culture mis aux essais. Le but de ce travail était d'identifier la proportion maximale de ce résidu (MCA) à incorporer à un substrat tourbeux afin de produire des plants de tomate de qualité. La valeur du compost (ou du méthacompost) peut varier en fonction de l'usage qui va en être fait et en fonction de l'effet qui est recherché par l'utilisateur [26]. Seul l'usage agronomique est envisagé lors de la présente étude.

Tableau 1. Identification et composition de mélanges confectionnés

\begin{tabular}{lcccccc}
\hline \multicolumn{3}{c}{ Mélanges T + MCA } & \multicolumn{5}{c}{ Mélanges T + MCA + CSB } \\
\hline Substrats & $\%$ T & $\%$ MCA & Substrats & $\%$ T & \% MCA & \% CSB \\
\hline SA & 100 & 00 & SA' & 80 & 10 & 10 \\
\hline SB & 90 & 10 & SB' & 70 & 20 & 10 \\
\hline SC & 80 & 20 & SC & 60 & 20 & 20 \\
\hline SD & 75 & 25 & SD & 50 & 20 & 30 \\
\hline
\end{tabular}

\subsection{Paramètres d'évaluation indirecte des substrats élaborés}

Au cours de ce travail, l'évaluation indirecte est basée surtout, d'une part, sur la détermination du pourcentage de germination de semences de tomate installées sur chaque substrat après une semaine de la levée, et d'autre part, sur le suivi de l'évolution de la partie aérienne des plants. 


\subsubsection{Suivi de la germination des semences}

La levée des plants ou germination des graines est une observation qui consiste à un comptage des plants levés. Elle permet de déterminer le taux de germination et la vitesse de la levée. Au niveau de chaque bloc, le \% des plants levés est calculé par rapport au nombre de graines de départ (12 alvéoles ou graines/bloc). Le pourcentage de germination correspond à la moyenne de trois blocs.

\subsubsection{Suivi de la croissance des plants en hauteur}

Pour suivre la croissance des plants, plusieurs paramètres peuvent être utilisés à savoir les paramètres morphologiques et les paramètres physiologiques. L'évaluation de l'impact des différentes combinaisons de MCA et CSB avec la tourbe sur la croissance des plants, est effectuée après quatre semaines de la date du semis. Elle est basée particulièrement sur l'étude des paramètres morphologiques des plants. Il s'agit donc de suivre l'accroissement en hauteur. Le suivi de l'évolution de la partie aérienne des plants a été conduit à partir des mesures (à intervalles plus ou moins réguliers de quatre jours), depuis le collet jusqu'au bourgeon apical à l'aide d'une règle. Lors de chaque suivi et pour chaque bloc, on a effectué des mesures selon un échantillonnage systématique non destructif. En effet, dans chaque bloc, on a choisi 5 plants homogènes : 5 plants x 3 blocs $=15$ plants/substrat, soit 75 plants de tomate/suivi/essai.

\subsubsection{Ajustement de la courbe de croissance des plants}

L'évolution de la croissance au cours du temps peut être traduite par des courbes de croissance, qu'il est utile de réduire à des modèles mathématiques simples, permettant de dégager des paramètres facilitant les comparaisons. Grossièrement, les courbes représentatives de la longueur de la tige sont des courbes en forme $\mathrm{S}$ dites sigmoïdes (en forme de sigma grec de mot, très allongé). L'établissement des courbes de croissance a été réalisé à l'aide du logiciel « ORIGIN, version $7.0 »$.

Si l'on néglige les variations de détails, les courbes de croissance peuvent être décrites par un modèle simple, qui conduit à des formules mathématiques commodes facilitant les comparaisons entre les divers matériels ou conditions expérimentales influençant la croissance. Particulièrement, l'évolution de la croissance en hauteur (y) est ajustée par un modèle sigmoïdal (Fit Boltzmann) ayant l'équation suivante : 


$$
y=\frac{A 1-A 2}{1+e^{(x-x 0) / d x}}+A 2
$$

Pour chaque essai, on a testé la variation de différents paramètres de cette équation (A1, A2, x0 et $\mathrm{dx}$ ) suivant le facteur contrôlé dans chaque essai (substrat).

\subsection{Méthodologie d'évaluation indirecte et dispositifs expérimentaux mis en place}

L'élevage des plants a pour objectif d'étudier l'incidence de la composition des substrats confectionnés sur la croissance et le développement des plants de tomate. En effet, l'évaluation de la croissance du végétal par l'estimation de l'ancrage des organes d'absorption des nutriments permet de définir le support adéquat. L'appréciation de la valeur agronomique du MCA, en état de mélange avec la tourbe et/ou CSB comme substrat de culture, s'est manifestée à l'aide de deux tests portant sur le semis des semences de tomate.

L'essai I s'est basé sur une substitution partielle de la tourbe par le MCA, alors que l'essai II a concerné une substitution partielle de la tourbe par le MCA et le CSB dans la confection des substrats de culture pour la production hors sol des plants de tomate. Le dispositif expérimental adopté lors de ces deux essais est le dispositif Blocs Aléatoires Complets (BAC) avec un seul facteur étudié (substrat de culture) et un seul facteur contrôlé (3 blocs). La disposition des substrats, lors de ces deux essais, est présentée sur les Figures 2 et 3 ci-après.

\begin{tabular}{|l|c|c|c|c|c|}
\hline Bloc I & SA & SB & SC & SD & SE \\
\hline Bloc II & SD & SC & SA & SE & SB \\
\hline Bloc III & SB & SD & SE & SC & SA \\
\hline
\end{tabular}

Fig.2. Dispositif expérimental adopté lors de l'Essai I

\begin{tabular}{|l|c|c|c|c|c|}
\hline Bloc I & SA' $^{\prime}$ & SB' $^{\prime}$ & SC' $^{\prime}$ & SD' & SE' \\
\hline Bloc II & SD' & SC' $^{\prime}$ & SA' $^{\prime}$ & SE' & SB' \\
\hline Bloc III & SB' & SD' & SE' & SC' & SA' $^{\prime}$ \\
\hline
\end{tabular}

Fig.3. Dispositif expérimental adopté lors de l'Essai II

\subsection{Analyse statistique des résultats relevés}

Les données obtenues pour chaque paramètre (germination, croissance en hauteur des plants) ont été interprétées en utilisant le logiciel statistique SPSS.17. L'interprétation fait appel 
respectivement à l'analyse de la variance (ANOVA) et à la comparaison des moyennes de différents types de traitement (Test Duncan) tout en recherchant là où les moyennes sont considérées comme étant égales, si au contraire, il y a une différence significative, le test « Duncan » permet de compléter l'interprétation et d'identifier les groupes de moyennes homogènes.

\section{RÉSULTATS ET DISCUSSION}

\subsection{Essai I : Substitution de la tourbe par le méthacompost avicole}

\subsection{1. Évolution de la germination des semences de tomate}

L'incorporation partielle du MCA, dans la confection des substrats de culture à base de tourbe, peut avoir une incidence sur le taux de germination des semences de tomate installées sur les divers substrats de cet essai. La Figure 4 ci-après illustre les résultats moyens relevés de la germination le long de la période du suivi.

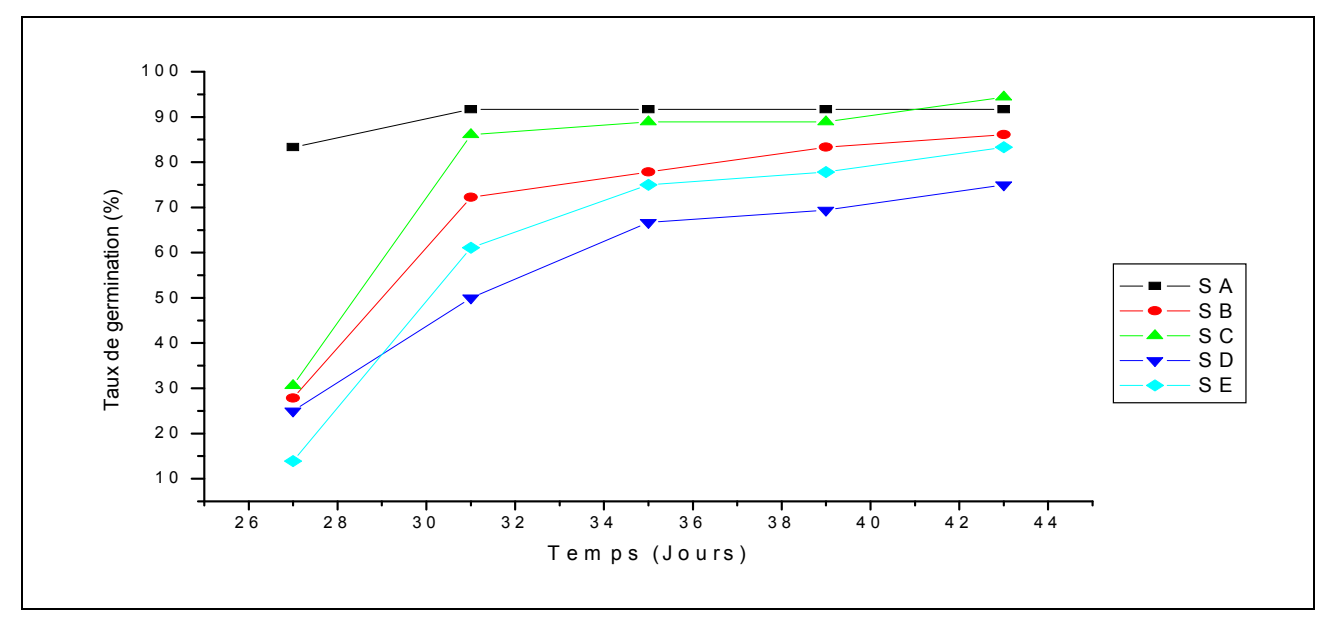

Fig.4. Pourcentages cumulés moyens de germination des semences de tomate

Le pourcentage de germination des semences de tomate sur les différents substrats peut constituer un critère d'évaluation surtout que les substrats utilisés présentent des pourcentages différents. La tourbe conserve le même taux de germination tout le long de l'essai, alors que les autres substrats confectionnés, ils présentent une évolution au cours du temps. En effet, le substrat SC est le meilleur mélange avec un taux de germination très élevé de l'ordre de 94,4\% dépassant même celui de la tourbe. La variation de la composition du mélange a un effet considérable sur la germination des plants de tomate. Il semble que la phytotoxicité et la faible 
Pa des substrats ont des incidences directes sur la germination. La phytotoxicité peut résulter de plusieurs facteurs comme le manque de l'oxygène dû à l'activité microbienne élevée, l'accumulation des composés toxiques (acides organiques), la concentration élevée en ammoniaque et la présence des métaux lourds et des sels minéraux (Epstein, 1997 cité par [27]). La plupart de ces facteurs influence simultanément sur la germination des graines et il est difficile par la suite de mettre en évidence le paramètre déterminant la plus grande incidence [27].

La maturité d'un compost est le plus souvent associée à la germination et la croissance des plants incluant la phytotoxicité, l'immobilisation de l'azote et l'équilibre des nutriments $[28,29]$. Les faibles taux de germination peuvent être expliqués donc par la présence de substances toxiques qui inhibent la germination des semences de tomate. En effet, les divers substrats ont enregistré des très faibles taux de germination par comparaison avec la tourbe (SA) surtout le long de deux premières semaines après début de la germination des semences sur tourbe. Il s'agit donc d'un retard remarquable dans la germination des graines de tomate sur les divers substrats. Ce retard est de l'ordre de 10 jours dans le cas de SC et peut atteindre 16 jours dans le cas de SE. Ce retard se réduit considérablement au cours du temps.

Selon Verdonck (1988) cité par [30], les substrats de culture devraient avoir un pH légèrement acide $(5,2$ à 6,3$)$. Les substrats $\mathrm{SD}$ et SE présentent des valeurs du $\mathrm{pH}$ qui dépassent légèrement la valeur maximale exigée $(6,3)$. Des répercussions négatives ont eu lieu sur la germination et l'émergence des semences (Castillo et al., 2004 cités par [30]) sur ces deux substrats.

\subsection{2. Évolution de la croissance en hauteur et survie des plants de tomate}

La hauteur exprimée en cm, constitue un bon indicateur de la capacité photosynthétique et de la surface de transpiration qui est corrélée avec le nombre des feuilles (Armson et Sadreika, 1974 cités par [31]). Le comportement des plants s'est montré variable d'un substrat à un autre et d'une observation de croissance à une autre. Lorsque les plants sont produits sur SB ou SC, l'indice de croissance augmente linéairement, en fonction de la proportion d'incorporation du MCA dans le mélange (Figure 5). Cet indice varie de façon quadratique lorsque les plants sont produits sur SD ou SE. Les résultats obtenus, concernant ces deux derniers substrats, peuvent être expliqués par les valeurs élevées de la masse volumique apparente (mva). Ce paramètre 
augmente la résistance mécanique du substrat qui a des conséquences directes sur la croissance et la morphologie des racines [31].

L'incorporation du MCA ne doit pas dépasser donc 20\%, au-delà de cette substitution il ya un retard remarquable de croissance. Les faibles indices de croissance et le retard par comparaison à la tourbe sont dus à la présence des substances toxiques inhibitrices de croissance. Selon Juste et al. [32], les métaux lourds, par exemple, affectent préférentiellement les systèmes racinaires. Des comptages de plantules en fonction du temps ou à une date donnée, des mesures de hauteur, de biomasse produite permettront d'évaluer ces différents symptômes.

Selon Juste et al. [32], les symptômes de phytotoxicité peuvent affecter la plante entière ou seulement une partie. La phytotoxicité peut être à l'origine du retard de croissance et/ou de développement. Ce point regroupe l'inhibition de la germination, le retard à la levée ou le retard de la croissance, les modifications phénologiques telles que le retard de floraison, le retard de nouaison. Ces types de manifestations sont les plus évidentes et les plus fréquentes.

On peut dire que l'intégration partielle du MCA dans la confection des substrats de culture pour la production des plants maraîchers est déconseillée, vu les résultats non encourageants obtenus dans nos conditions expérimentales.

Les substrats tourbeux usuels sont microbiologiquement inactifs. De ce fait, un agent pathogène rentrant en contact avec de tels substrats, il peut s'y développer rapidement et causer d'importants dégâts [33]. Selon Juste et al. [32], parmi les symptômes de la phytotoxicité des modifications de la composition du végétal sans manifestation externe. Ces modifications de la composition traduisent bien un trouble du fonctionnement normal de la plante et la cause doit donc être qualifiée de toxique. 


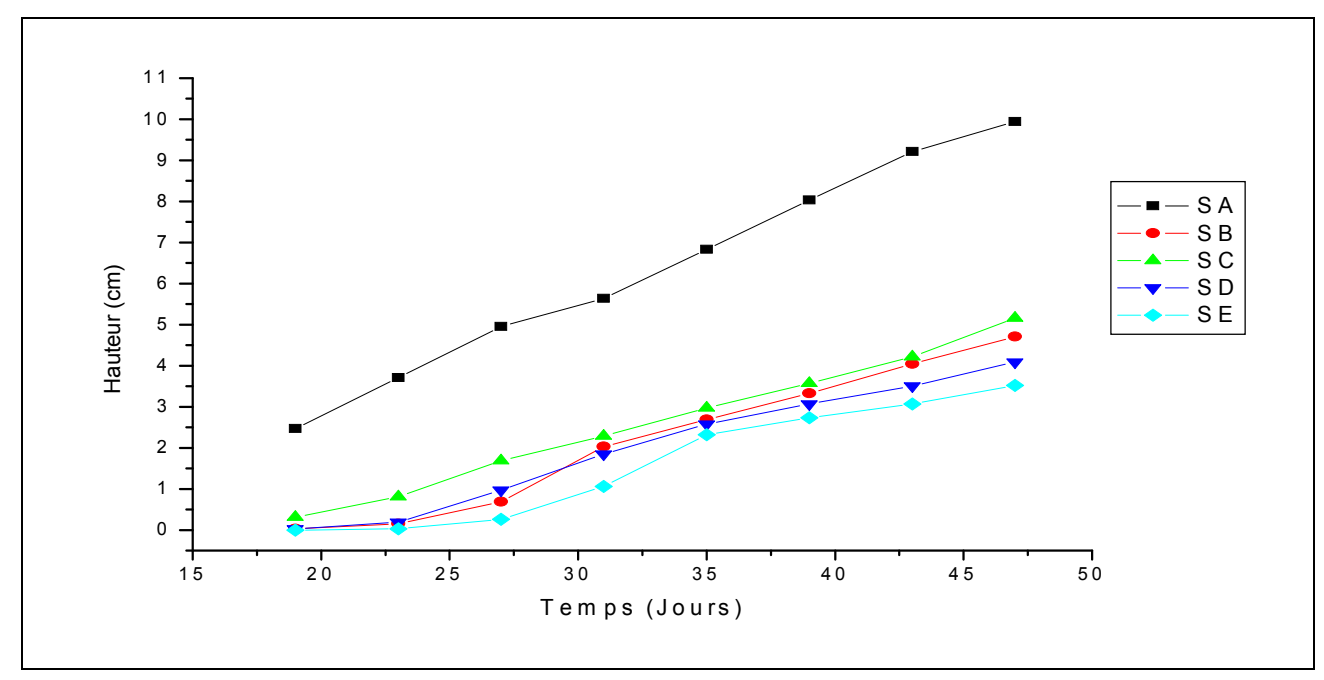

Fig.5. Évolution de la croissance moyenne en hauteur des plants de tomate (Essai I)

Les résultats de l'évaluation indirecte du MCA en mélange avec la tourbe sont moins encourageants que ceux obtenus pour le cas du méthacompost bovin (MCB) [36, 34, 8]. Les résultats relatifs au $\mathrm{MCB}[36,8]$ prouvent que la croissance en hauteur des plants du piment est quasi-identique pour les plants installés sur tourbe que sur MCB pur. Malgré que ses propriétés physiques ne soient pas tout à fait satisfaisantes, le MCB convient à être utilisé comme substrat de culture à l'état pur, mais il est préférable de l'incorporer avec la tourbe à raison de $60 \%$.

Les valeurs du $\mathrm{pH}$ (à l'exception de $\mathrm{SE}$ ) et de la $\mathrm{CE}$ déterminées sont acceptables pour un substrat de culture. Les brûlures affectant les plants installés sur SE et surtout les feuillages sont dues dans ce cas à la valeur élevée du $\mathrm{pH}$ [35].

L'incidence de la substitution partielle de la tourbe par le MCA, sur la croissance en hauteur des plants de tomate, est très variable par rapport à celle de la tourbe mélangée avec du MCB à raison de $60 \%$ [36]. Ce dernier donne une croissance plus rapide et des hauteurs des plants plus élevées (par comparaison à la hauteur moyenne des plants de piment installés sur la tourbe seule) sans présenter des anomalies végétatives.

Selon Comtois et Légaré [35], il faut considérer deux éléments complémentaires lorsque l'on veut comprendre les facteurs responsables de la croissance des plantes et l'impact de ceux-ci sur l'équilibre requis par les végétaux : Le substrat et l'eau. Selon Michel [37], les propriétés de stockage et surtout de circulation de l'eau et de l'oxygène dans les supports de culture horticoles présentent des fortes particularités, essentiellement liées au volume réduit de substrat 
(pot, conteneur). Ces volumes limités, disponibles pour le développement et le fonctionnement du système racinaire, créent des conditions de croissances très spécifiques pour la plante où les biodisponibilités hydrique et gazeuse fluctuent fortement et rapidement au cours de la journée mais aussi tout au long de la culture. Les conditions nutritives dans la zone racinaire peuvent devenir, par conséquent, très rapidement différentes des besoins réels et instantanés de la plante.

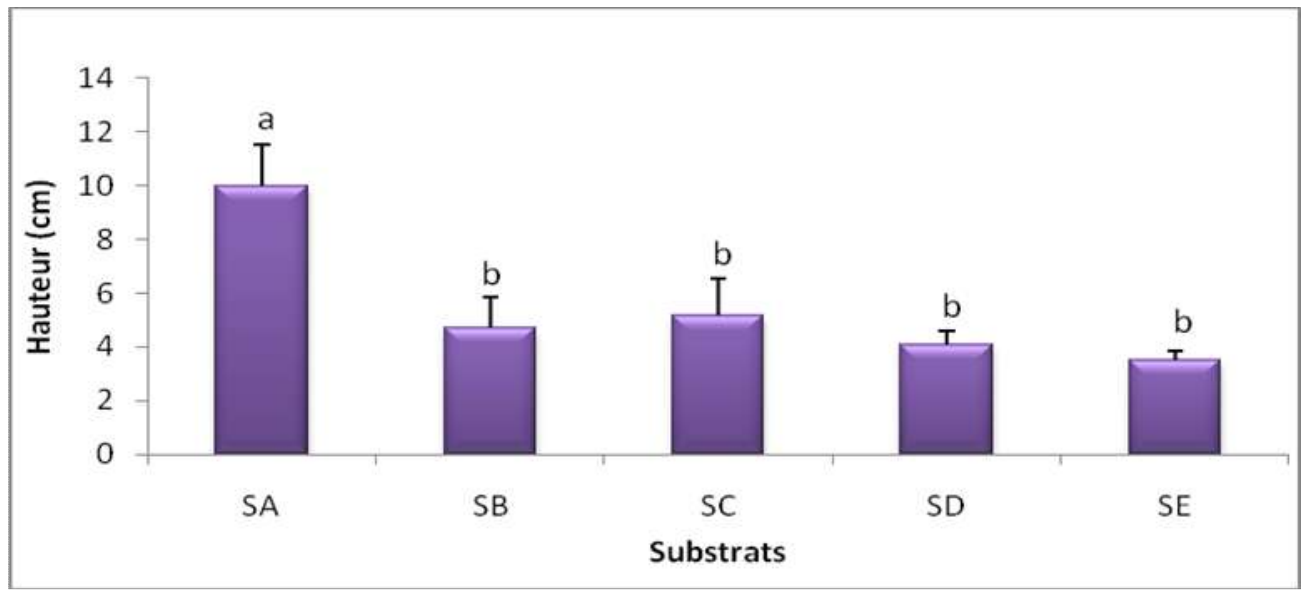

Fig.6. Variation de la hauteur moyenne des plants de tomate (Essai I)

L'analyse statistique s'intéressant uniquement à la dernière mesure de croissance (Figure 6), peut nous renseigner sur le résultat global généralement visé. D’après les résultats obtenus, il n'existe pas de différence significative entre les différents substrats en mélange. Ces résultats montrent aussi que l'incorporation de MCA à raison de 10, 20, 25 ou 30\% n'a pas d'effet considérable sur la croissance, au contraire, son incorporation est à l'origine d'une différence significative en croissance végétative par rapport aux plants installés sur tourbe.

Afin d'optimiser les apports d'intrants aux besoins de la plante, tout en limitant les rejets vers l'environnement, il est encore nécessaire d'améliorer notre compréhension de la dynamique de circulation de l'eau et de l'oxygène dans les substrats [38]. Et cela implique de répondre à la question suivante : comment évoluent les biodisponibilités en eau et en oxygène dans les supports de culture, au voisinage des racines et au cours du temps ? Pour répondre à cette question, il est nécessaire de développer une approche expérimentale afin de caractériser et d'analyser très précisément plusieurs propriétés (physiques, hydriques, physico-chimiques et structurales) participant aux processus de transfert de l'eau et de l'oxygène dans trois matériaux 
organiques modèles (tourbe brune largement usagée en pépinières horticoles, ressources organiques locales MCA et CSB testés et/ou utilisés en pépinières forestières modernes).

Les plants installés sur SB et SE ont présenté comme pour le cas de quelques plants installés sur MCB seul des tiges grêles, sensibles et quelques unes ont été brûlées et finissent par flétrir. Le pourcentage d'altération le plus élevé est enregistré pour le cas des plants installés sur SE. Il est de l'ordre de 6,6\% (Figure 7).

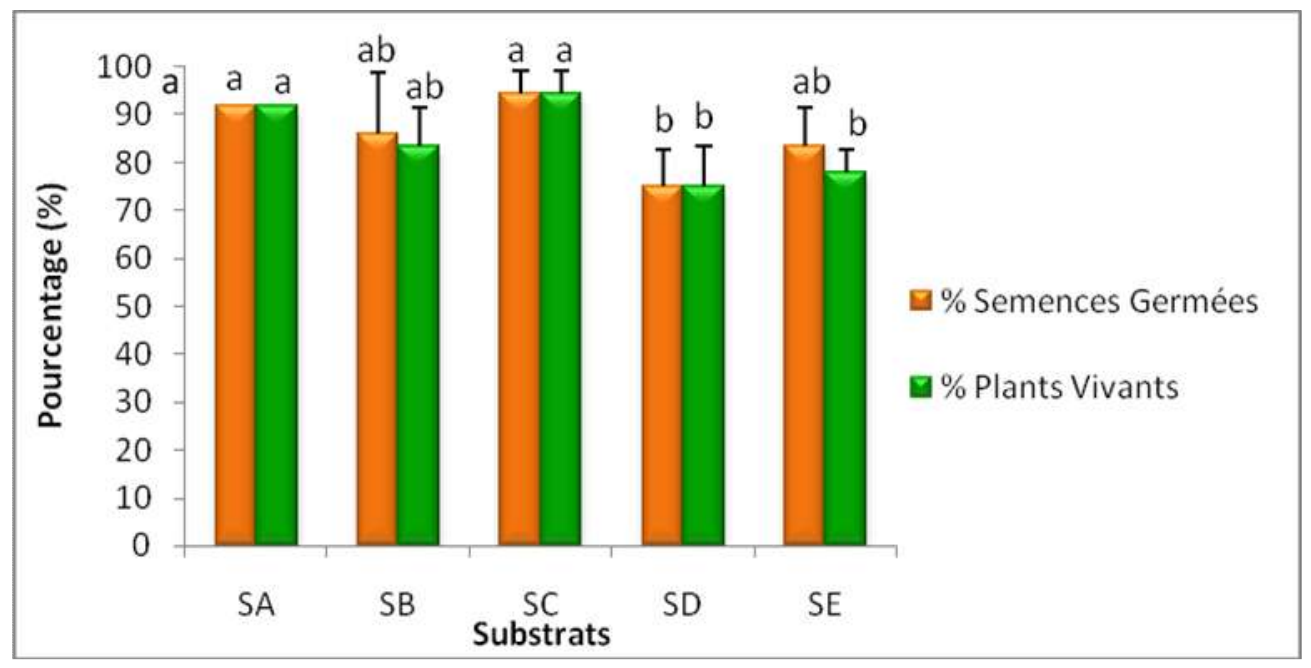

Fig.7. Incidence de la composition du mélange sur la survie des plants de tomate (Essai I)

Selon Heller [39] une aération insuffisante des racines nuit fortement à l'absorption de l'eau et des sels minéraux. L'asphyxie des racines perturbe le catabolisme respiratoire, les tissus fermentent puis meurent. Cependant, les racines présentent un hydrotropisme positif mais uniquement pour l'eau courante et aérée [40].

On doit tenir compte aussi de la carence vraie (réelle). En effet, chaque élément a ses symptômes de carence qui varient d'une espèce à une autre. Il peut y avoir encore une confusion entre symptôme de carence et les autres contraintes que causent les brûlures, l'excès d'humidité ou de température, les maladies cryptogamiques et les ravageurs de culture.

\subsubsection{Résultats de l'ajustement de la croissance par une sigmoïde}

Les résultats de l'ajustement de la croissance en hauteur des plants de tomate installés sur les substrats de cet essai sont présentés dans le Tableau 2. 
Tableau 2. Variation de différents paramètres de la sigmoïde

\begin{tabular}{lccccc}
\hline Substrats & $\mathbf{R}^{2}$ & $\mathbf{A 1}$ & $\mathbf{A 2}$ & $\mathbf{x 0}$ & $\mathbf{d x}$ \\
\hline SA & 0,99702 & $-28,46220$ & 29,08018 & 11,10963 & 50,96933 \\
\hline SB & 0,98959 & $-0,736290$ & 5,29620 & 33,45136 & 6,641400 \\
\hline SC & 0,99714 & $-17,69064$ & 61,29899 & 126,88370 & 88,31480 \\
\hline SD & 0,99366 & $-0,808200$ & 4,451850 & 31,425350 & 6,775050 \\
\hline SE & 0,99095 & $-0,108570$ & 3,363300 & 33,033710 & 3,188960 \\
\hline
\end{tabular}

La qualité de l'ajustement est meilleure dans le cas des plants installés sur SC $\left(\mathrm{R}^{2}\right.$ la plus élevée) ayant pour courbe d'ajustement celle présentée par la Figure 8. Il s'agit d'une courbe de croissance plutôt linéaire que sigmoïde.

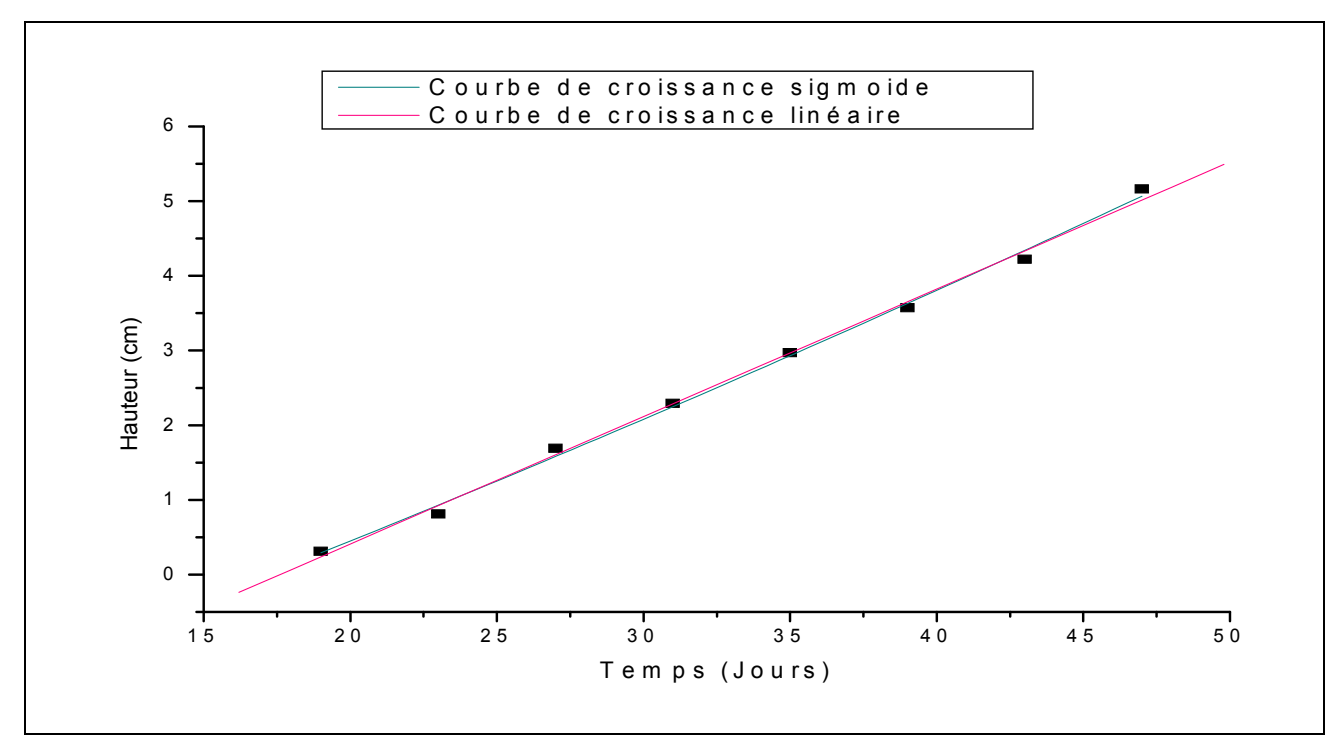

Fig.8. Ajustement de la croissance moyenne des plants de tomate (Essai I)

\subsection{Essai II: Substitution de la tourbe par le méthacompost avicole et le compost sylvicole}

\subsubsection{Taux de germination}

La qualité des méthacomposts, des composts et des substrats en mélange peut être définie comme étant l'ensemble de leurs caractéristiques qui leurs donnent une valeur et qui assurent la possibilité de leur usage sans nuisances pour l'environnement ou les cultures produites sur eux. L'incidence de l'incorporation partielle du MCA et du CSB dans la confection des substrats de culture peut être déduite à partir de la Figure 9 ci-après qui illustre la variation des pourcentages de germination des plants de tomate en fonction du substrat. Le taux élevé de 
germination est l'un des critères de qualité des substrats de culture. Dans ce cadre, il est à noter que la plupart des substrats confectionnés présentent des taux de germination élevés. Les \% enregistrés les plus importants sont relatifs à SC' $(91,7 \%)$ et aux substrats SA' et SD' $(83,3 \%)$. Les substrats $\mathrm{SB}^{\prime}$ et $\mathrm{SE}$ ' ayant les \% de germination les plus faibles constituent, d'après les résultats statistiques observés, un seul groupe.

Zucconi et De Bertoldi (1987) cités par [29] ont discuté les différences entre les essais de germination et les essais de croissance. Les essais de germination fournissent une image instantanée de phytotoxicité, tandis que les essais de croissance seront affectés en continu des changements de la stabilité ou de la maturité du compost examiné : il peut y avoir des effets préjudiciables, en partie, sur la croissance, mais des effets bénéfiques plus tard.

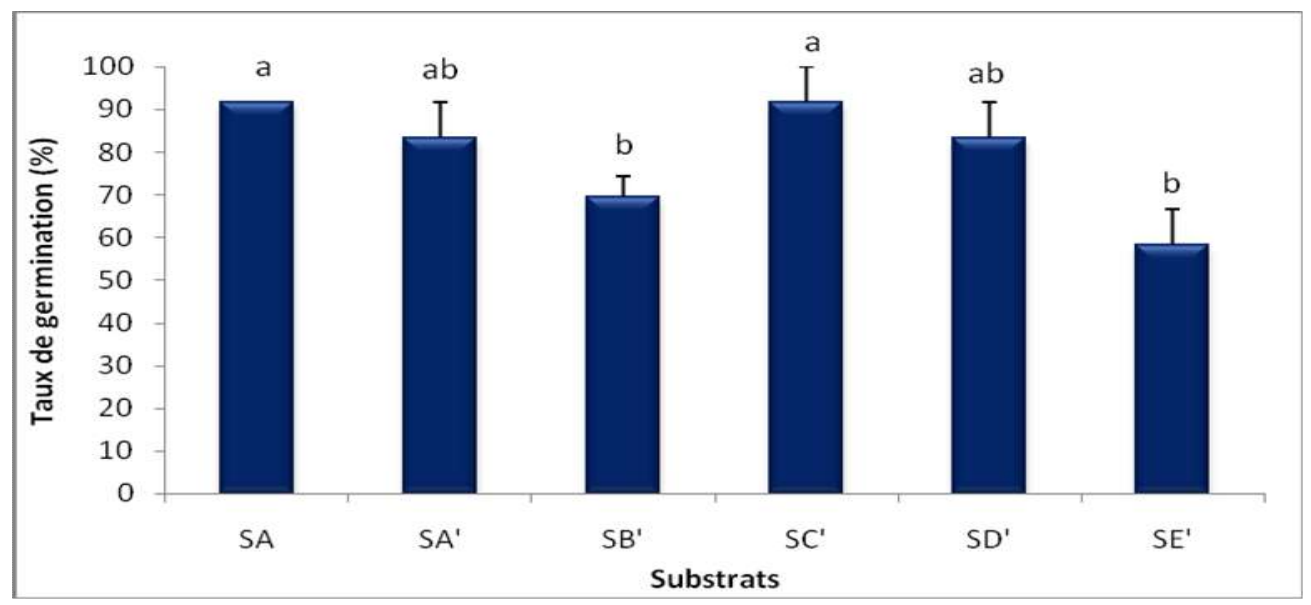

Fig.9. Effet du mélange sur la variation de la germination des graines de tomate (Essai II)

\subsection{2. Évolution de la croissance des plants en hauteur}

Les résultats du suivi de croissance en hauteur des plants de tomate au cours du temps sont présentés par la Figure 10. Selon Gras [41], la porosité, particulièrement, est l'un des facteurs physiques les plus importants qui affectent la croissance. Elle a un effet sur les activités biologiques (fonctionnement des racines : aération, température) et la disponibilité des éléments nutritifs (alimentation en eau du végétal).

La plupart des auteurs admettent que les risques de manifestations des effets phytotoxiques sont liés à l'état de maturité des composts. Selon Alidadi et al. [42], l'apport de ces MO insuffisamment stabilisées est susceptible d'affecter le développement de la végétation (mauvaise germination, retard ou déficience de croissance). Ce phénomène peut être attribué à 
divers facteurs : éléments toxiques présents dans les matières initiales et imparfaitement dégradés, métabolites produits dans les premiers stades de la décomposition (ammoniac, acides organiques volatils), immobilisation de l'azote dans le sol (ou substrat de culture) aux dépens de la plante (Blanco, 1996 cité par [23]), anoxie pour les racines résultant d'une importante consommation d'oxygène par les microorganismes minéralisateurs.

En plus du retard de germination, la composition des substrats confectionnés permet de réduire davantage la croissance en hauteur des plants de tomate. L'utilisation du MCA, pour la production des plants maraîchers au stade pépinière, a des répercussions négatives sur la croissance en hauteur des plants qui a varié d'une façon quadratique et elle vient de diminuer d'une façon très considérable à partir d'une incorporation de $20 \%$ du MCA. On vient de remarquer qu'il est plus bénéfique d'incorporer le MCA et le CSB dans les mêmes proportions (cas des substrats $\mathrm{SA}^{\prime}$ et $\mathrm{SC}$ '). Les résultats relevés montrent encore qu'un faible apport de 10\% de ces deux matériaux (MCA et/ou CSB) a donné des plants sains et de meilleure qualité par rapport aux autres mélanges. Il convient de constater que la MO, en l'occurrence, le MCA ou le CSB, agissent sur les plants par ses effets directs (disponibilité des éléments nutritifs) et par ses effets indirects en tamponnant le substrat tourbeux (protection des plants contre les agents pathogènes). À cet égard, ce ratio de mélange pourrait être conseillé en pépinières maraîchères, mais avec beaucoup de précautions.

La tourbe est la matière la plus usagée dans les productions horticoles. Elle est cependant utilisée en mélange avec d'autres matériaux pour rendre le substrat plus performant et adapté à la production choisie. Sur le plan qualité chimique, le MCA pourrait être incorporé à la tourbe puisque certains avantages suite à son emploi ont été démontrés au cours de la présente investigation. Des recherches supplémentaires sont nécessaires afin d'évaluer l'effet de cette composante sur la croissance d'un plus grand nombre d'espèces maraîchères. Il semble que les espèces répondent différemment à l'incorporation du MCA dans les substrats de culture tourbeux. 


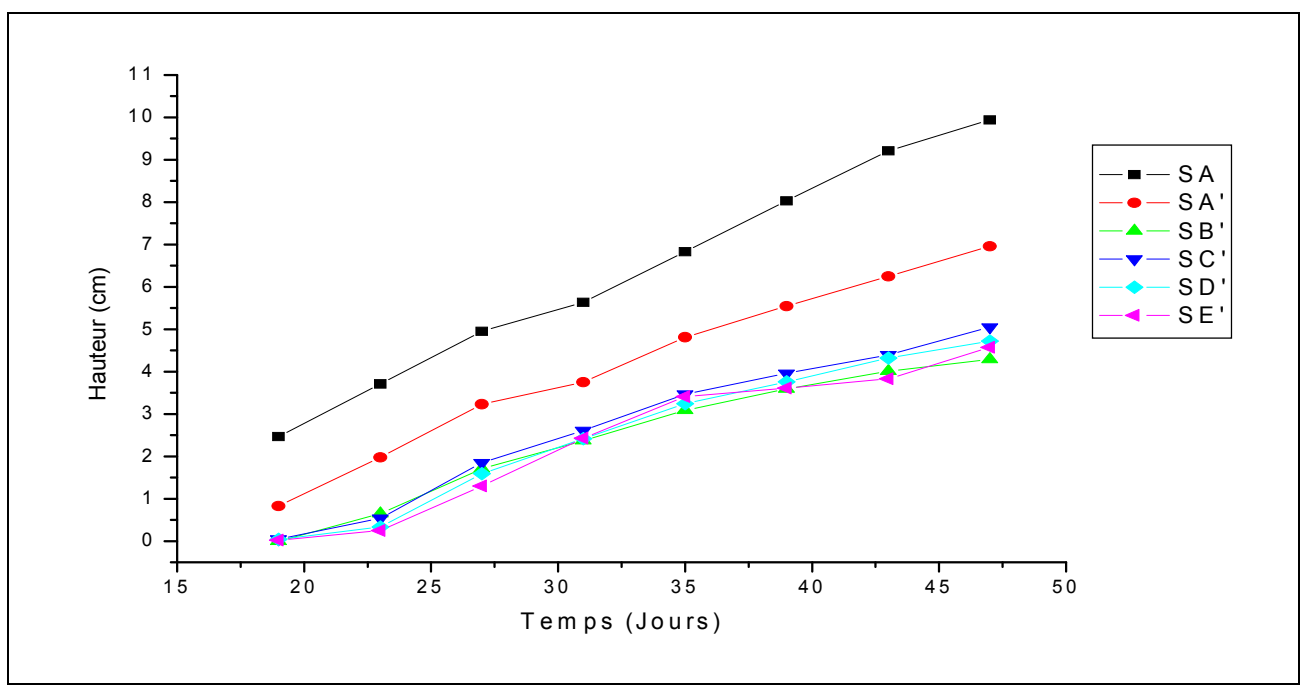

Fig.10. Évolution de la croissance moyenne en hauteur des plants de tomate (Essai II)

L'analyse statistique intéresse la dernière mesure de croissance. Elle nous renseigne sur le résultat global généralement visé. Les résultats de la comparaison des moyennes sont donnés directement sur la Figure 11 ci-après.

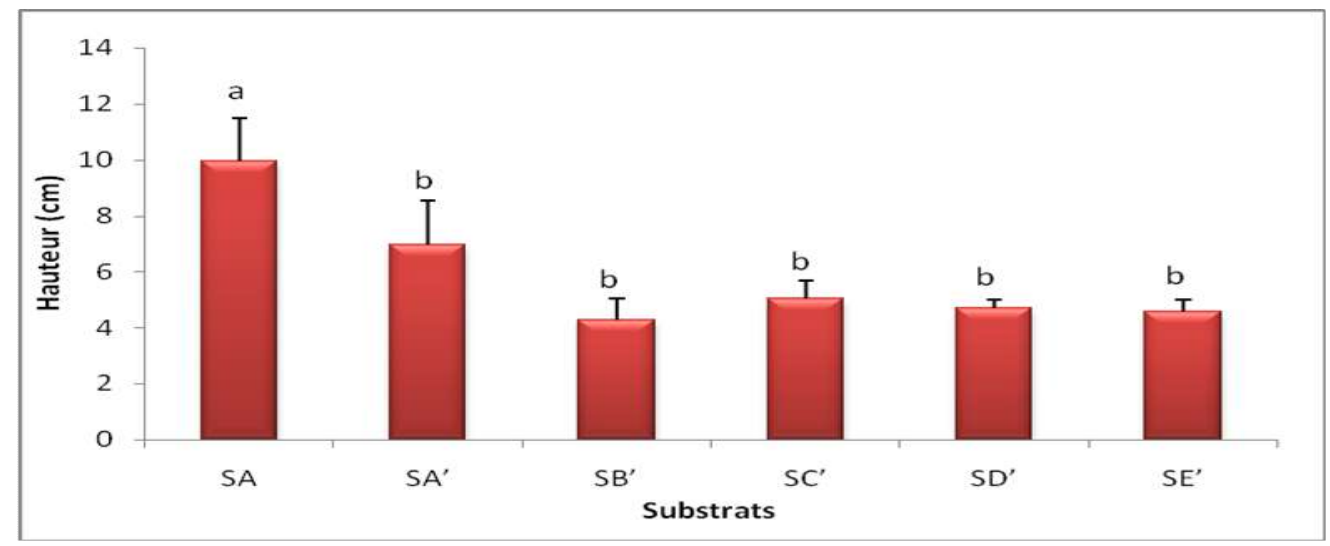

Fig.11. Variation de la hauteur moyenne des plants de tomate de (Essai II)

Les plants de tomate installés sur tourbe sont les meilleurs de point de vue croissance en hauteur en comparaison avec ceux installés sur les autres substrats à base de mélange. À ce propos, on a observé une stimulation de la croissance en hauteur et une augmentation plus ou moins importante du nombre de feuilles. Le développement le plus faible est relevé sur SB' (Figure 12).

Les incorporations du MCA et du CSB ont une répercussion négative sur la croissance des organes végétatifs. Les hauteurs considérées des plants sont moins élevées que celles du témoin. L'émission de nouvelles feuilles a été également réduite ainsi que leurs tailles. 
Il est à noter que ces résultats sont souvent en relation avec les caractéristiques chimiques et physiques des substrats. Les teneurs élevées en éléments nutritifs ( $\mathrm{N}, \mathrm{Ca}, \mathrm{K}, \mathrm{Na})$ peuvent causer une phytotoxicité des jeunes plants. Les faibles teneurs en air peuvent causer une asphyxie qui permet la diminution de la croissance des jeunes plants, comme elles peuvent causer le flétrissement de ces derniers avec quelques cas de mortalité des plants installés sur SA' et SE'.

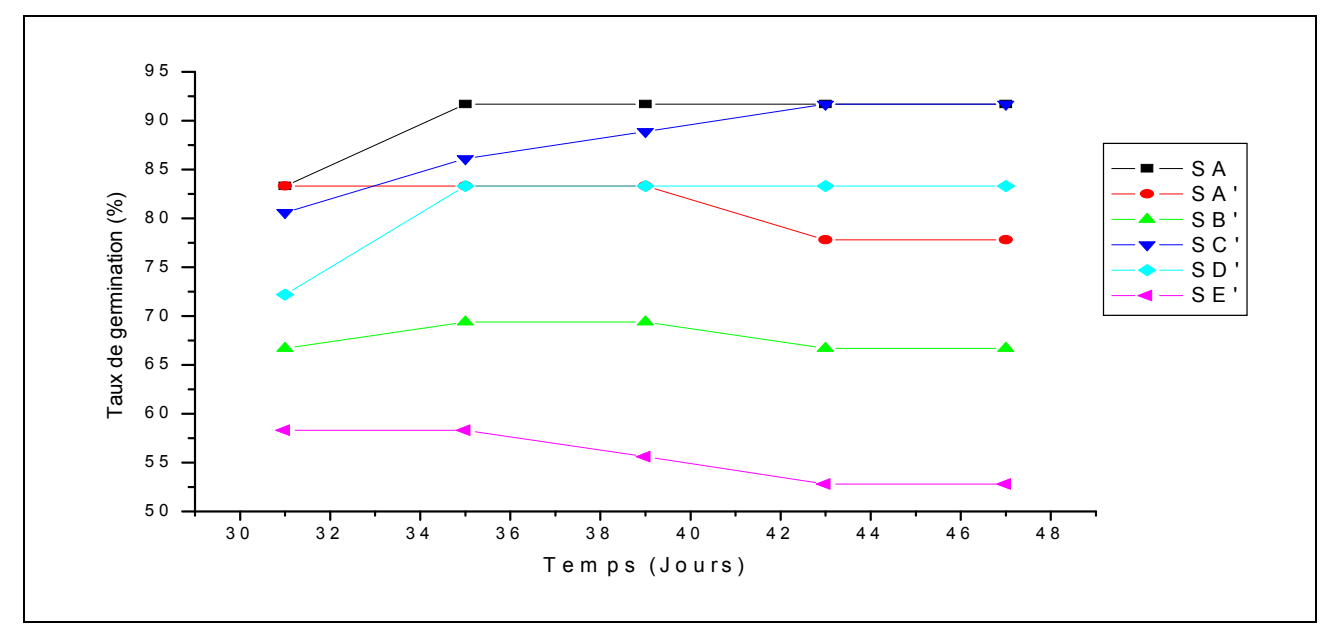

Fig.12. Effet de la composition des mélanges sur la survie des plants de tomate (Essai II)

\subsubsection{Résultats de l'ajustement par une sigmoïde de la croissance}

Les résultats de l'ajustement de la croissance en hauteur des plants installés sur les substrats de cet essai sont présentés dans le Tableau 3.

Tableau 3. Variation de différents paramètres de la sigmoïde

\begin{tabular}{lccccc}
\hline Substrats & $\mathbf{R}^{\mathbf{2}}$ & $\mathbf{A 1}$ & $\mathbf{A 2}$ & $\mathbf{x 0}$ & $\mathbf{d x}$ \\
\hline SA $^{\prime}$ & 0,99708 & $-63,01934$ & 12,55896 & $-39,16377$ & 34,22747 \\
\hline SB' $^{\prime}$ & 0,99844 & $-2,362345$ & 4,77449 & 25,12875 & 8,43915 \\
\hline SC' $^{\prime}$ & 0,99347 & $-2,77156$ & 5,73848 & 26,04736 & 9,32149 \\
\hline SD' $^{\prime}$ & 0,99409 & $-1,29999$ & 5,98101 & 28,92882 & 6,89106 \\
\hline SE' & 0,98521 & $-0,56128$ & 4,33813 & 29,24701 & 4,54615 \\
\hline
\end{tabular}

La courbe d'ajustement, présentée sur la Figure 13 est relative au cas des plants de tomate installés sur SB' ayant la meilleure qualité d'ajustement. Il s'agit d'une courbe de croissance sigmoïde. D'après Wacquant [43], de telles courbes concaves vers le haut (dy/dt croissant, donc $\mathrm{d} 2 \mathrm{y} / \mathrm{dt} 2>0)$, puis après un point d'inflexion $(\mathrm{d} 2 \mathrm{y} / \mathrm{dt} 2=0)$, concave vers le bas $(\mathrm{d} 2 \mathrm{y} / \mathrm{dt} 2<0)$, 
sont dites sigmoïdes (en forme de sigma grec de mot, très allongé). Ceci montre que la vitesse de croissance est d'abord relativement lente puis s'accélère, passe par un maximum au niveau du point d'inflexion, et enfin diminue jusqu'à ce que la longueur ou le diamètre soient sensiblement constants. Le passage de la phase linéaire à la partie concave de la courbe (courbe sigmoïde) implique un ralentissement, dû à un ensemble de facteurs de décélération qui peuvent avoir plusieurs causes. La plus importante, dans le cas de l'organisme végétal, est la difficulté grandissante de l'approvisionnement en substances nutritives de la plante dans son ensemble et de chacun de ces organes ainsi que de l'appauvrissement du substrat au voisinage des racines et des distances de plus en plus longues à parcourir entre les organes absorbants, les organes assimilateurs et les lieux d'utilisation. Il s'ajoute des phénomènes de toxicité qui tiennent à la sénescence des tissus de la plante.

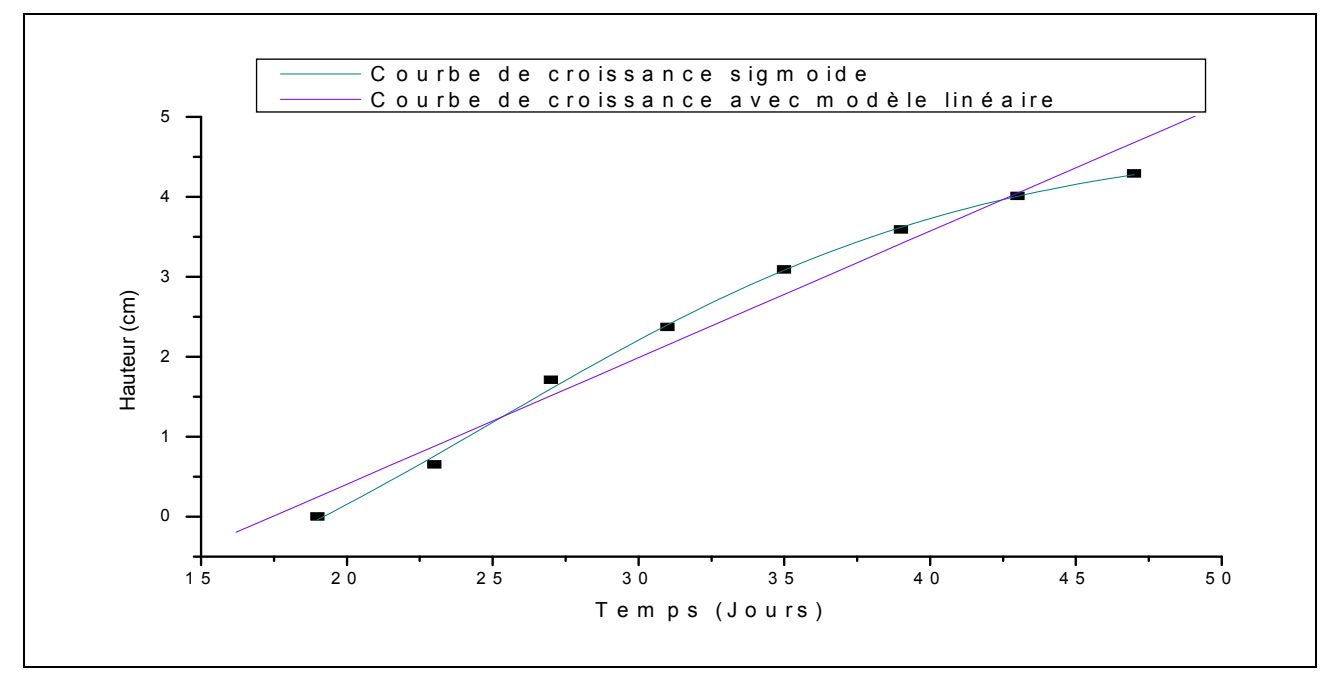

Fig.13. Ajustement de la croissance moyenne des plants de tomate (Essai II)

\section{CONCLUSION}

La biométhanisation de la biomasse animale (fientes avicoles seules) et/ou le compostage de la biomasse végétale (broyats sylvicoles) établissent des options de remplacement face à l'emploi massif de la tourbe importée. La substitution partielle de la tourbe par le méthacompost avicole (issu du bassin de décantation d'un digesteur industriel) dans la confection des substrats de croissance des plants maraîchers, constitue l'un des objectifs recherchés lors de cette étude. L'objectif primordial étant l'appréciation indirecte de l'incidence du méthacompost avicole (MCA) sans/avec ajout du compost sylvicole brut (CSB), en tant que substitut partiel de la 
tourbe importée, sur le comportement agronomique hors sol des plants de tomate, à savoir : germination, accroissement en hauteur et survie des plants, ajustement de la croissance.

Les résultats essentiels obtenus, à partir de deux essais accomplis, révèlent une bonne germination des semences de tomate sur les mélanges ( $80 \%$ Tourbe $+20 \%$ MCA) et $(60 \%$ Tourbe $+20 \% \mathrm{MCA}+20 \% \mathrm{CSB})$ dépassant même celle sur Tourbe, une croissance en hauteur la plus élevée enregistrée pour ces mêmes mélanges incorporant 20\% MCA. En outre, le flétrissement le plus élevé est observé pour le cas des plants installés sur le mélange (70\% Tourbe $+30 \%$ MCA) avec un taux presque de $7 \%$. La qualité de l'ajustement est meilleure dans le cas des plants installés sur le substrat $(80 \%$ Tourbe $+20 \%$ MCA) ayant une courbe de croissance plutôt linéaire que sigmoïde. La courbe d'ajustement relative au cas des plants de tomate installés sur le mélange $(70 \%$ Tourbe $+20 \% \mathrm{MCA}+10 \% \mathrm{CSB})$ ayant une courbe de croissance sigmoïde pour la meilleure qualité d'ajustement relevée. Dans l'ensemble, le mélange $(80 \%$ Tourbe $+10 \% \mathrm{MCA}+10 \% \mathrm{CSB})$ semble être le meilleur substrat à préconiser pour produire des plants maraîchers de qualité, tout en limitant les importations de tourbe.

\section{RÉFÉRENCES}

[1] Demirbas A., Energy Conversion and Management, 2001, 42 (11), pp. 1357-1378.

[2] Gijzen H., Water Science and Technology, 2002, 45 (10), pp. 321-328.

[3] Tou I., Igoud S., Touzi A., Revue des Energies Renouvelables, N Spécial : Biomasse, Production et Valorisation, 2001, pp. 103-108.

[4] M'Sadak Y., Ben M'Barek A., Zoghlami R.I., Baraket S., Revue des Energies Renouvelables, 2011, 14 (2), pp. 343-356.

[5] M’Sadak Y., Ben M'Barek A., Baraket S., Revue Nature \& Technologie, 2012, 7, pp. 81-86.

[6] M'Sadak Y., Zoghlami R.I., Algerian Journal of Arid Environment (AJAE), 2012, 2 (2), pp. 16-27.

[7] M'Sadak Y., Ben M’Barek A., Zoghlami R.I., Revue Nature \& Technologie, 2013, 8 (C), pp. 19-26. 
[8] M'Sadak Y., Ben M'Barek A., International Journal of Innovation and Applied Studies, 2013, 4 (2), pp. 343-352.

[9] Verrier D., Morfaux J.N., Albagnac G., Touzel J.P., The French programme on methane fermentation. Biomass, 2 (1), 1982, pp. 17-28.

[10] Beline F., Gac A., La méthanisation: un moyen de valoriser la matière organique des déjections animales et de réduire les émissions de gaz à effet de serre. SINFOTECH- Les Fiches Savoir-faire, CEMAGREF, 2007, pp. 4.

[11] Macias-Corral M., Samani Z., Hanson A., Smith G., Funk P., Yu H., Longworth J., Bioresource Technology, 2008, 99, pp. 8288-8293.

[12] Oost JFr., De Toffoli M., Estimation de la valeur fertilisante de digestats issus de la biométhanisation. Résultat de 3 années d'expérimentation en culture de maïs (2009 à 2011), Centre Pilote de Maïs, Belgique, 2011, pp. 5.

[13] Muller F., Zdanevitch I., Qualité des composts et des digestats. Colloque National Prévention \& Gestion des déchets dans les territoires, Paris, France, 2013, pp. 8.

[14] Mignon Ch., Utilisation du digestat comme fertilisant en agriculture. Valbiom, Belgique, 2009, pp. 15.

[15] Martel S., Desmeules X., Landry Ch., Lavallée S., Paré M., Tremblay F., Valeur fertilisante des digestats de méthanisation. Recherche et Innovation en Agriculture (AGRINOVA), Canada, 2013, pp. 3.

[16] Moral R., Paredes C., Bustamante M.A., Marhuenda-Egea F., Bernal M.P., Bioresource Technology, 2009, 100, pp. 5454-5460.

[17] Fuchs J.G., Galli U., Schleiss K., Wellinger A., Caractéristiques de qualité des composts et des digestats provenant du traitement des déchets organiques. Association Suisse des Installations de Compostage (ASIC) \& Forum Biogaz Suisse, 2001, pp. 26.

[18] Mbuligwe S.E., Kassenga G.R., Resources, Conservation and Recycling, 2004, 42, pp. 183-203.

[19] Poeschl M., Ward S.H., Owende P.H., Renewable and Sustainable Energy Reviews, 2010, 14 (7), pp. 1782-1797. 
[20] Amigun B., Von Blottnitz H., Energy Conversion and Management, 2007, 48, pp. 3090-3094.

[21] Pouech PH., Intérêt des digestats et possibilités de valorisation. Ateliers d'échange sur les aspects techniques et réglementaires de la méthanisation de la biomasse, 2007, pp. 14.

[22] Gauthier F., Gagnon S., Dansereau B., Can. J. Plant Sci., 1998, 78, pp. 131-138.

[23] Van Der Gaag D.J., Van Noort F.R., Stapel-Cuijpers L.H.M., Kreij C., Termorshuizen A.J., Van Rijn E., Zmora-Nahum S., Chen Y., Scientia Horticulturae, 2007, 114 (4), pp. 289-297.

[24] Sellami-Brini F., Compostage de déchets organiques : Effet du compost sur la qualité physico-chimique des terreaux en pépinière et sur les caractéristiques pédo-géochimiques en arboriculture. Doctorat en Sciences géologiques, Faculté des Sciences de Sfax, Tunisie, 2009, pp. 222.

[25] ALCOR, AXENNE, Étude stratégique pour le développement des énergies renouvelables en Tunisie- Bilan des réalisations. Rapport final de l'Agence Nationale des Énergies Renouvelables (ANER), 2003, pp. 148-157.

[26] Houot S., Compostage, qualité des déchets entrants et qualité des produits finis. Recyclage des déchets organiques en agriculture biologique. Bergerie Nationale de Rambouillet et Guide des Matières Organiques. Journées Techniques ITAB-Bergerie Nationale, France, 2001, pp. 23-24.

[27] Tiquia S.M., Chemosphere, 2010, 79, pp. 506-512.

[28] Bernal M.P., Paredes C., Sanchez-Monedero M.A., Cegarra J., Bioresource Technology, 1998, 63, pp. 91-99.

[29] Bernal M.P., Alburquerque J.A., Moral R., Bioresource Technology, 2009, 100, pp. 5444-5453.

[30] Kerkeni A., Contribution à la valorisation des composts et des jus de composts : Incidence sur la fertilisation et la protection phytosanitaire de quelques espèces légumières. Thèse Agriculture Durable pour l'obtention du Diplôme de Doctorat en Sciences Agronomiques de 1'Institut Supérieur Agronomique de Chott Mariem (ISA-CM), Tunisie, 2008, pp. 158. 
[31] Lamhamdi M.S., Fortinn J.A., Ammari Y., Ben Jalloun S., Poirier M., Fecteau B., Bougacha A., Godin L., Évaluation des composts, des substrats et de qualité des plants élevés en conteneurs. Rapport technique : Exécution des travaux d'aménagement de trois pépinières pilotes en Tunisie. Publication DGF de Tunisie et Pampev Internationale Ltée, Québec, Canada, 1997, pp. 121.

[32] Juste C., Solda P., Dureau P., Test agronomique simple destiné à juger rapidement de la phytotoxicité éventuelle et du degré de maturité d'un compost d'ordures ménagères. Chapitre d'ouvrage édité - BMA : Utilisation agricole des déchets. Résultats de dix années de recherches. Comité "Sols et Déchets" 1973-1983. Document élaboré par l'Agence Nationale pour la Récupération et l'Élimination des Déchets (ANRED), Nantes, France, 1985, pp. 4.

[33] Fuchs J.G., Bieri M., Nouveaux biotests pour caractériser la qualité des composts. AGRAR-Forscbung, 2000, 7 (7), pp. 314-319.

[34] M’Sadak Y., Ben M’Barek A., Tayachi L., Revue Nature \& Technologie, 2012, 6, pp. $59-70$.

[35] Comtois M., Légaré M., La fertilisation des plantes ligneuses cultivées en contenant. Programme Horti-2002, Direction de 1'Innovation Scientifique et Technologique, 2004, pp. 57.

[36] M'Sadak Y., Ben M'Barek A., Baraket S., Agronomic interest of the residues of rural biomethanisation applied to the bovine biomass. Displays, ISOFAR /MOAN Symposium: Soil Fertility and Crop Nutrition Management in Mediterranean Organic Agriculture, March 2010, Sousse, Tunisia.

[37] Michel J.Ch., Les propriétés physiques des tourbes : Une qualité majeure à leur utilisation comme support de culture. Sciences Agronomiques Appliquées à 1'Horticulture (SAGAH). INRA-INH, Université d'Angers, France, sd, pp. 7.

[38] Ecobichon M., Substrats. Lettre d'Information de Falienor-Terreaux de France, 2006, 17, pp. 6 .

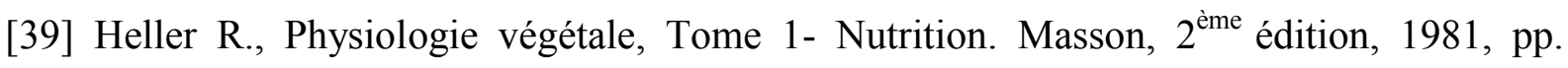
$117-120$. 
[40] Heller R., Physiologie végétale, Tome 2- Développement. Masson, 2ème édition, 1982, pp. $150-156$.

[41] Gras R., Rev. Sc. Sol, 1981, pp. 171-186.

[42] Alidadi H., Parvaresh A.R., Shahmansouri M.R., Pourmoghadas H., Iran. J., Environ. Health. Sci. Eng., 2008, 5 (2), pp. 137-140.

[43] Wacquant C., Maîtrise de la conduite climatique de la tomate sous serre et abris en sol et hors sol. Éditions du Centre Technique Interprofessionnel des Fruits et des Légumes (CTIFL), France, 1995, pp. 127.

\section{How to cite this article:}

M'Sadak Y. and Ben M'Barek A. Valorization agricultural of a solid digestate avicolous resulting from the industrial biomethanisation in Tunisia. J. Fundam. Appl. Sci., 2015, 7(3), 298-321. 